\title{
Attrition und Osmokinetik - Zwei Konzepte zur Pathogenese des Trockenen Auges
}

\author{
Gysbert-Botho van Setten (D)
}

Eingegangen: 31. März 2021 / Angenommen: 21. September 2021 / Online publiziert: 8. Oktober 2021

(C) Der/die Autor(en) 2021

\begin{abstract}
Zusammenfassung Die neuen Erkenntnisse der Pathophysiolgie des Trockenen Auges erkennen das Zusammenspiel von Tränen, Augenoberfläche und Lidoberfläche als eine funktionelle Einheit an. Der Begriff der Benetzungsfähigkeit der Tränen in Abhängigkeit der mikrotektonischen Anatomie der Augenoberfläche relativiert die Anforderungen an Träne und Tränenersatzmittel. Das Model der Attrition, welches die Effekte der friktionsneutralisierenden Kapazität des Tränenfilms, der Reibung und die Bedeutung der Mechanotransduktionskapazität des Epithels zusammenfasst, wird eingeführt und dessen pathophysiologische Bedeutung erläutert. Attrition und Benetzung bestimmen zusammen grundlegende pathophysiologische Vorgänge in der Augenoberfläche wie Aktivierung von Nerven (subjektive Beschwerden) sowie Entzündung und beeinflussen damit die Dynamik der Pathophysiologie, und den Übergang von vorübergehenden Beschwerden zu einer manifesten Erkrankung des Trockenen Auges. Die Betrachtung der Osmolarität als numerischer statischer Grenzwert zur alleinigen Diagnose des Trockenen Auges ist klinisch nicht haltbar. Das neue, dynamische Model der Osmokinetik, zeigt dagegen eine Alternative auf, in der die Tageschwankungen und die Beachtung des durchschnittlichen Osmolaritätsniveaus größere Bedeutung gewinnen und damit der eigentlichen pathophysiologischen Bedeutung der Osmolarität gerechter wird.
\end{abstract}

Vorbehalt Alle angegebenen Modelle und Hypothesen des Autors sind, bis auf Weiteres keine Anleitung oder Empfehlungen zu Behandlungen. Der Autor übernimmt keine Verantwortung für jedwede Behandlung, die auf den wiedergegebenen Modellen basieren könnten.

Ass. Prof. Dr. Dr. G.-B. van Setten, MD ( $\triangle)$

Dept. of Clinical Neuroscience, Division of Eye and Vision,

Lab of DOHF and Wound Healing, Karolinska Institutet,

Eugeniavägen 12/level 6, 17104 Solna, Schweden

gysbert.van.setten@ki.se
Schlüsselwörter Osmolarität · Erkrankung des Trockenen Auges · Attrition · Osmotischer Stress . Neurogene Entzündung

\section{Attrition and osmokinetics - Two concepts for the pathogenesis of dry eye disease}

Summary The new findings of the pathophysiology of the dry eye recognize the interplay of tears, the surface of the eye and the surface of the eyelid as a functional unit. The concept of the wettability of tears in relation to the micro-tectonic anatomy of the surface of the eye relativizes the requirements for tears and tear substitutes. The model of attrition, which summarizes the effects of the mechano-transduction capacity of the epithelium and the friction-neutralizing capacity of the tear film: the principle of this model is introduced and its pathophysiological significance explained. Attrition and wetting together determine fundamental pathophysiological processes in the surface of the eye such as activation of nerves (subjective complaints) as well as initiation and maintenance of inflammation and thus influence the dynamics of the pathophysiology and the transition from temporary symptoms to the dry eye as a disease. The consideration of osmolarity as a numerical static limit value for the sole diagnosis of dry eye is not clinically tenable. The new, dynamic model of osmokinetics, on the other hand, shows an alternative in which the daily fluctuations and the observation of the average osmolarity level gain greater importance and thus do better justice to the actual pathophysiological significance of osmolarity and its effect on ocular surface stress.

Keywords Osmolarity · Dry eye disease - Attrition · Osmotic stress · Neurogenic inflammation 


\section{Einleitung}

In den letzten Jahren ist das Beschwerdenbild des Trockenen Auges immer mehr in das zentrale Tätigkeitsgebiet der Augenärzte gerückt. In zunehmenden $\mathrm{Ma}$ e werden unangenehme Benetzungsstörungen nach Operationen [1-4] und Strahlenbehandlungen [5] auch klinisch ernster genommen und behandelt [6]. Auch ist man sich der wirtschaftlichen Bedeutung einer angemessenen und effektiven Behandlung mehr als je zuvor bewusst $[7,8]$. Gerade deshalb sind zeitgerechte Informationen über dieses Gebiet und seiner Veränderungen von großer Bedeutung. Ohne eine Kenntnis der neuesten Einblicke in die Pathogenese ist eine optimierte Behandlung nicht möglich.

Im Folgenden werden einige der wesentlichsten neuen Einblicke illustriert.

- Das Trockene Auge ist kein statischer Zustand, sondern eine, sich in Entwicklung befindliche Erkrankung

- Die Pathogenese des Trockenen Auges hat Vorstufen, sogenannte Prodromalstadien

- Das Trockene Auges kann ein vorübergehender Zustand oder eine progressive Erkrankung sein

- Eine strikte Einteilung in spezifische Defizienzformen ist klinisch selten möglich

- Klinische Zeichen der Augenoberfläche haben nur eine relative Aussagekraft

- Tränenfilmosmolarität kann kein „Goldstandard“ sein, ein exakter Grenzwert ist unwahrscheinlich

- Osmokinetische Modelle mit Beachtung des osmotischen Stresses ersetzen statische Modelle

- Friktion und Reibung als Hauptmediatoren in der Pathogenese, der Begriff der Attrition

\section{Hintergrund und Einsichten}

Die Grundlage einer erfolgreichen Behandlung des Trockenen Auges ist die Erfassung und Charakterisierung einer vorliegenden Benetzungsstörung als „Schmierdefizits“ an der Augenoberfläche. Allein das Vorliegen eines Schmierdefizites ist jedoch nicht gleichbedeutend mit der Diagnose einer Benetzungsstörung mit Krankheitswert, d.h. eines klinisch signifikanten Trockenen Auges. Dieser Unterschied ist aus diagnostischer Sicht, der Klassifizierung der beobachteten Veränderungen, und der Therapie sowie auch aus administrativer, also aus verwaltungstechnischer Sicht (korrekter ICD-Code), unter gegebenen Umständen individuell von großer Bedeutung. Es gibt einen sehr subtilen Übergang von präklinischen, sensations-orientierten Austrocknungsbeschwerden zu einer klinisch sichtbaren Erkrankung des Trockenen Auges. Eine Phase mit alleinigen, wenn auch typischen Beschwerden ohne pathomorphe Veränderungen geht also einer späteren Phase mit auch klinisch sichtbaren Veränderungen voraus. Solche Veränderungen können sehr subtil sein; zum Beispiel kann die BUT stark reduziert sein, auch wenn keine An- färbung vorhanden ist („Short BUT-DE“). Es handelt sich trotzdem um ein Trockenes Auge. Es muss daran erinnert und betont werden, dass das Auftreten von Benetzungsdefiziten an der Augenoberfläche von der ausreichenden Schmierfähigkeit des Tränenfilms, der die Augenoberfläche bedeckt, abhängt sowie auch von der viskoelastischen Dissipationskapazität in Bezug auf die ständig ausgeübten mechanischen Herausforderungen durch die sich bewegenden Lider [9]. Die viskoelastische Dissipationskapazität erfasst das Vermögen des Epithels durch normale Schichtung und Hydratationszustand externe mechanische Käfte und Scherkräfte zu absorbieren oder/und zu verteilen.

Der aktuelle Stand der Pathophysiologie des multifaktoriellen Systems des Trockenen Auges und eine Zusammenfassung der Vielzahl von Untersuchungsergebnissen wurde zuletzt in Dry Eye Workshop II dargestellt [10]. Es ist jedoch ein komplexes System, indem die Hauptursache für ein Trockenes Auge in den Tränen selbst, aber auch an/in den Oberflächen lokalisiert sein kann. Dass die Tränenmenge als solche und die Zusammensetzung der Tränen jedoch nicht allein pathophysiologisch für die Manifestation von Schmierdefiziten des Auges verantwortlich sein kann, zeigt sich insbesondere beim Vorhandensein des anatomischen Trockenen Auges [11]. Als Anatomisches Trockenes Auge bezeichnet man das Vorhandensein von anatomischen Unterbrechungen der homogenen Oberflächentektonik der Hornhaut, die zu einem lolaliserten verfrühten Tränenfilmaufriss führt. In diesem Zustand reicht es aus, wenn eine der wichtigen Komponenten des Tränenfilms im Verhältnis zum Schmierbedarf der Augenoberfläche unzureichend oder mangelhaft ist. Das Alter scheint pathophysiologisch dabei eine wichtige Rolle zu spielen [12]. Bei dem Anatomischen Trockenen Auge führt das pathomorphologische Korrelat einer mikrotektonischen Veränderung der Oberflächenkontur zu einem lokalisierten verfrühten Tränenfilmaufriss (Break Up Time, BUT). In Anbetracht solcher mikrotektonischen Veränderungen der Oberflächenkontur, welche auch häufig nach Laseroperationen beobachtet werden können (Abb. 1), ist es offensichtlich, dass diese anatomischen Situationen von der Tränenflüssigkeit maximale Qualitäten erfordern, um eine adäquate Befeuchtung zu gewährleisten. Mit zunehmendem Alter kann jedoch davon ausgegangen werden, dass diese Ansprüche nicht mehr erfüllt werden können und es treten Beschwerden auf. Das Vorhandensein eines qualitativen Trockenen Auges muss dabei nicht unbedingt durch die Abwesenheit oder Verminderung von Komponenten gekennzeichnet sein; allein die pathologische Verschiebung der Zusammensetzung reicht aus. Dies ist zum Beispiel bei fehlerhaftem Vorkommen von Lipiden der Fall (Abb. 2), die eine Beteiligung der Meibom-Drüsen in der Pathophysiologie des Trockenen Auges wiederspiegelt [13].

Diese Differenzierung zwischen qualitativem und quantitativem Trockenen Auge muss berücksichtigt 


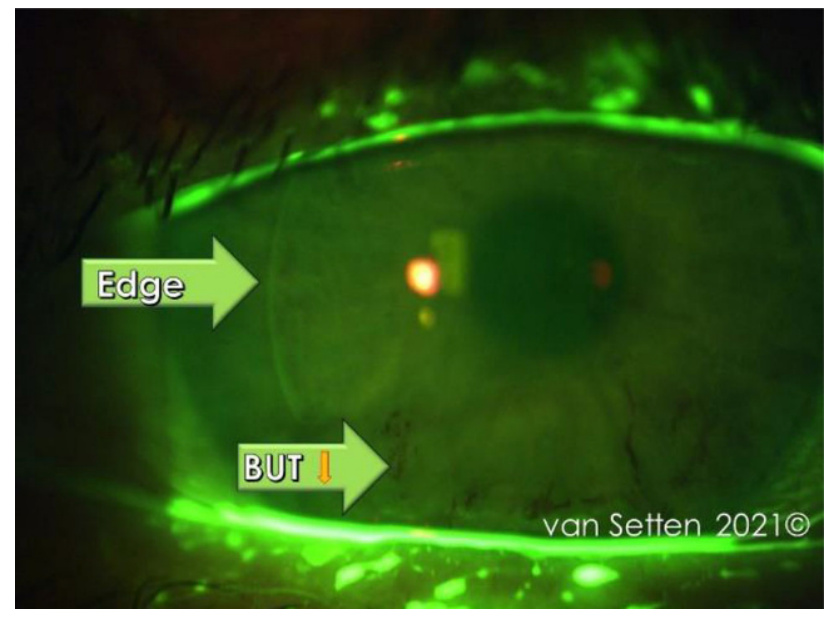

Abb. 1 Anatomisches Trockenes Auge nach Excimer-Laseroperation mit sichtbarer Kantenbildung (Edge) und mit lokalisiertem, vorzeitigen Tränenfilmaufriss (Tränenfilmaufreißzeit BUT)

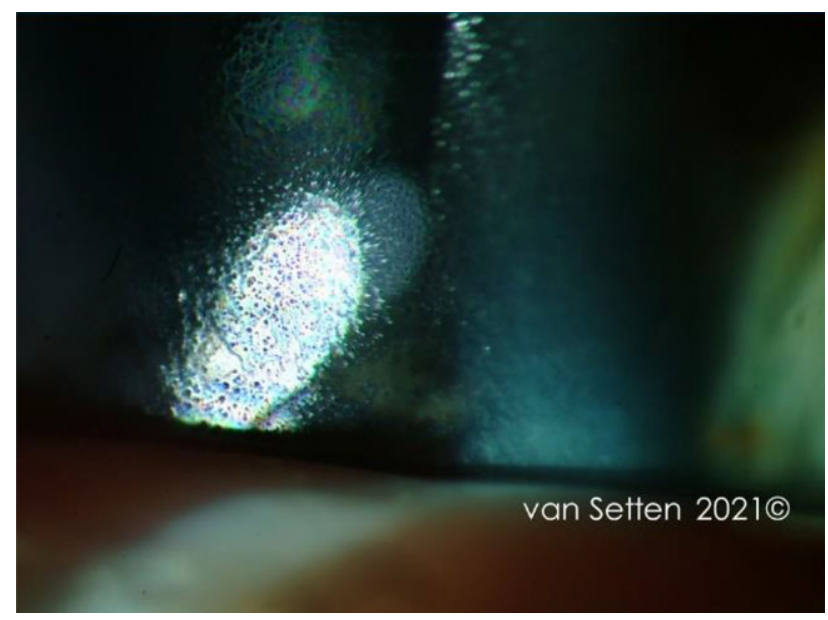

Abb. 2 Tränenfilm mit fehlerhafter Lipid-Zusammensetzung

werden, wenn therapeutische Richtlinien gegeben werden, wie kürzlich in den Nordischen Anleitungen für die Behandlung des Trockenen Auges [14]. Moderne Modelle betrachten eine solche klassische Trennung [15] jedoch als relativ und zeitabhängig (Abb. 3). Je nach Umgebung können sich die Eigenschaften im Laufe der Zeit ändern, was häufig zu einer gemischten Form der Erkrankung des Trockenen Auges führt. Da die Behandlung von Mischformen komplexer ist [16], gibt es einen guten Grund für eine effiziente Behandlung früherer Stadien, wo eine erfolgreiche allein substituierende Behandlung oft einfacher ist.

Die als Standard geltenden Vitalfärbungen der Hornhaut und Schleimhaut mit Fluoreszein [17] oder Lissamingrün [18] geben einen gewissen Aufschluss über den Schweregrad des Trockenen Auges (Abb. 4). Obgleich dabei die Anzahl und ungefähre Lage der Färbungen auf der Augenoberfläche sich im Allgemeinen nicht dramatisch in kurzer Zeit ändern, so

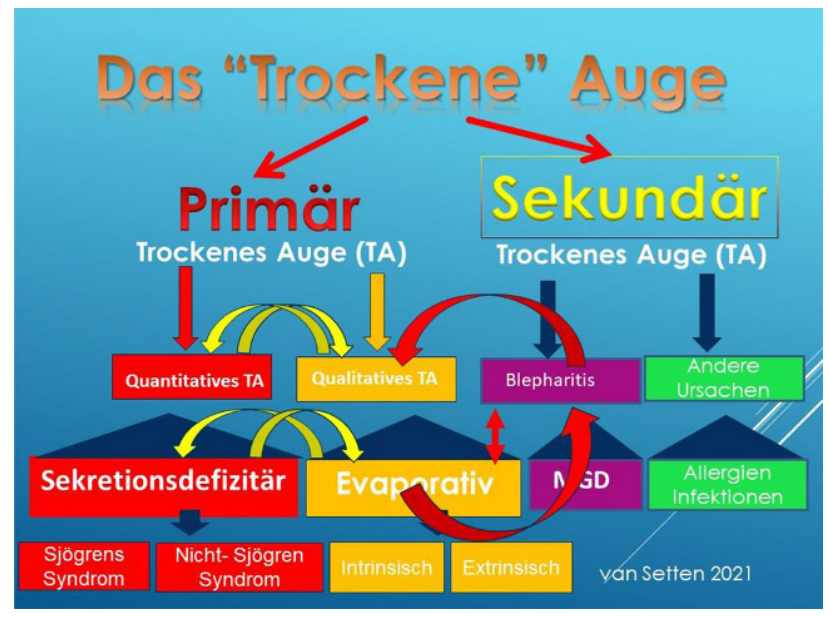

Abb. 3 Klassische Unterscheidung zwischen qualitativen und quantitativen Formen des primären Trockenen Auges und den sekundären Formen des Trockenen Auges sowie mögliche Übergänge im Laufe der Zeit

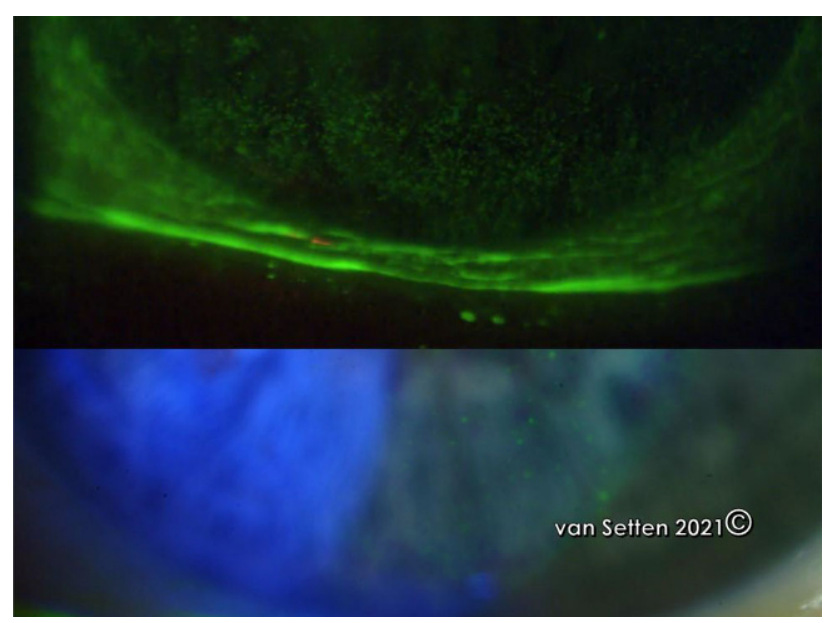

Abb. 4 Fluoreszein Färbung von leichteren (untere Bildhälfte) und schwereren Formen des Trockenen Auges (obere Bildhälfte mit stärkerer Färbung)

ist die exakte Position der Färbungen (,dots“) jedoch nicht stabil und unterliegt einer zeitabhängigen Verschiebung. Die Färbungen als solche spiegeln einen Situationszustand der oberen, externen Augenoberfläche wieder, was jedoch dem Geschehen in den tieferen Schichten der Augenoberfläche selbst nur bedingt entspricht. Eine kräftige Färbung der Augenoberfläche zeigt sicher einen bedeutenden Benetzungsdefekt auf, gibt jedoch nur indirekt und bedingt Aufschlüsse über die, in der Augenoberfläche ablaufenden pathophysiologischen Vorgänge, wie zum Beispiel Entzündungen.

Dennoch ist es allgemein akzeptiert, dass eine stärkere Anfärbung der Augenoberfläche mit erhöhten Entzündungsparametern einhergeht. Dabei gehören tiefer gelegene und intensive entzündliche Veränderungen in der Pathophysiologie des Trockenen Auges zu den fortgeschrittenen Stadien des Trockenen Auges $[13,19]$ und sind Gegenstand der Behandlung mit 


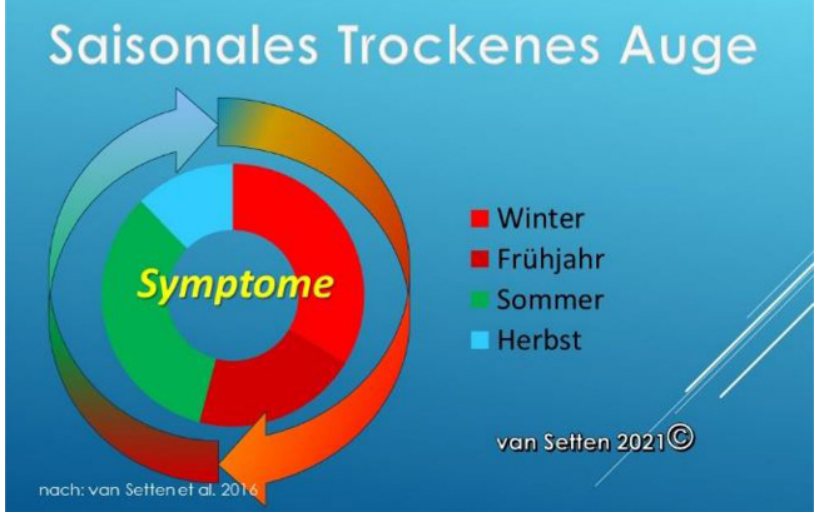

Abb. 5 Das jahreszeitliche (saisonale) Trockene Auge. Maximale Beschwerden in Winter (rot), weniger im Sommer (grün/ blau)

Immunomodulatoren wie Steroide, Cyclosporin A [20, 21] oder ICAM-1-Inhibitoren [22]. Die Anfänge in der Entwicklung des Trockenen Auges dagegen sind oft subtil und schleichend, vorrübergehende Prodromalstadien sind mehr von Symptomen als vom Vorliegen klinischer Befunde geprägt. Vorrübergehende Symptome als isolierte Ereignisse lassen eine problemlose „restititio ad integrum“ erwarten; eine gehäufte Wiederholung sollte jedoch als mögliches Prodromalstadium einer Krankheitsdisposition aufgefasst werden.

In der Pathophysiologie gibt es einen großen Unterschied zwischen der kontinuierlich fortschreitenden, sich ständig verschlechternden Erkrankung des Trockenen Auges und dem vorübergehenden, redundanten gelegentlichen Auftreten des Trockenen Auges (z.B. durch Umwelteinflüsse oder durch jahreszeitliche Schwankungen) [23]. In diesem Modell folgen symptomreiche Intervalle im Winter, gefolgt von eher symptomfreien Zeitintervallen im Sommer (Abb. 5). In der Pathogenese müssen diese Formen Trockener Augen als eine andere Entität betrachtet werden. Aufgrund ihres vorübergehenden Charakters können spezifische Risikofaktoren identifiziert und erfolgreich therapeutisch angegangen werden. Oft verschwinden Symptome von selbst und klinische Zeichen, wie die Fluoreszein-Färbung, sind entweder nicht vorhanden, oder nur mild und kurzfristig zu beobachten. Ausreichend früh erkannt, bieten diese Zustände auch die Chance für eine erfolgreiche Behandlung und damit sogar die therapeutische Möglichkeit, einen Übergang in eine chronische Phase $\mathrm{zu}$ verzögern oder sogar zu vermeiden. In dieselbe Kategorie gelegentlicher vorübergehender episodischer Beschwerden des Trockenen Auges, fallen auch schubweise verlaufende Formen, die als „dry eye disease flares“ bezeichnet wurden [24], wie sie unter bestimmten Umweltbedingungen vorrübergehend auftreten können.

Veränderungen an der Augenoberfläche bedeuten jedoch nicht nur sichtbare Zeichen einer Erkrankung der Oberfläche, sondern signalisieren auch pathophysiologische Geschehnisse unter der Oberfläche. Hier steuern die grundlegenden Mechanismen des Teufelskreises („vicious circle“) [13, 19] die Prozesse im Sinne einer kontinuierlichen Verschlechterung. In diesem intra- und subepithelialen Raum wird die Entwicklung der Pathophysiologie des Trockenen Auges bestimmt. Obwohl die grundlegenden Prozesse und Teilkomponenten in diesem Zirkel immer besser bekannt werden, berücksichtigt die zweidimensionale Betrachtungsweise nicht die dreidimensionale Entwicklung im Raum der Zeit. Eine unidirektionale Entwicklung mit der Perspektive einer kontinuierlichen Verschlechterung als vorhersehbarer Verlauf ohne Behandlung steht einer retrograden Entwicklung als Behandlungsresultat entgegen, also ein gegen gerichteter Vorgang, im Sinne einer ausgebremsten oder verzögerten Verschlechterung. Der therapeutische Zugang, mit der Absicht den Verlauf der Entwicklung $\mathrm{zu}$ verlangsamen, ist dabei sehr von aktualisierten Modellen der Pathophysiologie abhängig. Hier liegt nun ein verstärkter Fokus auf der Bedeutung der Benetzungskapazität der Tränenflüssigkeit und der mechanischen Transduktion, d.h. der Auswirkungen der (Ab-)Reibung [25] auf das darunter liegende Gewebe, auch als „Attrition“ bezeichnet [26].

In fortgeschrittenen Phasen des Trockenen $\mathrm{Au}-$ ges haben die Epithelzellen an Volumen verloren und werden immer größeren Scherkräften ausgesetzt. Hier sind die interzellulären Regulationsmechanismen größten Herausforderungen ausgesetzt. Mit zunehmendem Schweregrad und Dauer der epithelialen Exposition und den metabolischen Herausforderungen hat jeder zusätzliche Faktor eine exponentielle Bedeutung.

Hier sind an erster Stelle die osmotischen Herausforderungen zu nennen (auch als Osmostress bezeichnet), zusätzlich auch die Verstärkung der Mechanotransduktion durch einen Wegfall des epithelialen Kisseneffekts (physiologisch durch normale Schichtung sowie Hydratationszustandes des Epithels vorliegendes mechanisches Absorptionsvermögen des Epithels). Beide sind sehr eng mit der nervalen Signalgebung und der Steuerung der sekundären entzündlichen Prozesse verknüpft.

Gerade die Hyperosmolarität als solche wird in der Pathophysiologie des Trockenen Auges besonders hervorgehoben [10, 27, 28], auch wenn die Messungen in der Praxis nicht mit dem klinischen Bild korrelieren $[29,30]$. Um die Bedeutung der Osmolarität zu verstehen, ist es wichtig, von statischen Modellen mit Grenzwerten wegzukommen und dynamische Modelle zu erwägen. Statische Modelle basieren auf Grenzwerten. Im Falle der Osmolarität besteht die Hyperosmolarität also auf der Erhöhung der Osmolarität in der Tränenflüssigkeit über diesen Grenzwert. Hyperosmolarität als solche, ist ein bekannter Stressfaktor; Zellen sind jedoch adaptativ und können sich der Umgebung anpassen. Einer statischen Umgebung können 
sich Zellen aber viel leichter anpassen, als einer Umgebung mit großen und häufigen Schwankungen, welche die Anpassung der Zellen kontinuierlich und immer wiederholend herausfordert. Dies ist das Grundprinizp des Osmo-Stresses. Jedes Geschehen am Auge ist ja ein höchst dynamisches Ereignis. Sich von absoluten Werten, sogenannten Grenzwerten zu distanzieren (wie zum Beispiel $318 \mathrm{mOsmol} / \mathrm{L}$ ) [10] hat auch den klinisch diagnostischen Vorteil, dass beim Vergleich verschiedener Messungen des gleichen Parameters, z. B. der Osmolarität zu verschiedenen Zeitpunkten im Tagesverlauf bei bestmöglicher Standarisierung des Vorgehens auch wichtige Variationen erfasst werden können. Diese Variation als solche, sowohl in ihrem Wert als Tageschwankung als auch in Ihrer Dynamik wie Frequenz, Frequenzdichte etc [31] erlaubt den gemessenen Wert der einzelnen Messungen in Relationen zu den anderen Werten zu sehen und auch das durchschnittliche Niveau zu erfassen, auf welchem diese Veränderungen geschehen. Hierin liegt die Attraktivität des Models der Osmokinetik. Osmotischer Stress ist aus der Physiologie sehr gut bekannt und auch seine Auswirkungen auf den Zellmetabolismus [32, 33]. Als neuer Aspekt kommt hier die negative Auswirkung des osmotischen Stresses auf die Mitochondrien hinzu [34, 35].

Das pathogenetische Gewicht der Hyperosmolarität ist von der osmotischen Toleranz der Augenoberfläche abhängig, der Osmotoleranz, welche einer osmotischen Pufferfunktion gleichkommt. Diese auch als adaptive Stressantwort (,adaptive stress responses“) bezeichnete Reaktion von Zellen sind zum Überleben und Erhalt der Homeostase erforderlich [36] und resultieren in einer Verlangsamung verschiedener intrazellulärer Prozesse. Die Auswirkungen der Schwankungen der Osmolarität eines Mediums sind
Abb. 6 a Geringe pathophysiologische Bedeutung (Osmostress) von mäßigen Tagesschwankungen (DVO Daily Variation of Osmolarity) der Osmolarität bei im Durchschnitt normaler, d.h. im physiologischen Niveau liegender Osmolarität $(306 \mathrm{mOsmol} / \mathrm{L}$, blaue Linie). Die Farben der Pfeile spiegeln die maximalen (rot) und minimalen (grün) Werte einer einzelnen Schwankung wieder. b Größere pathophysiologische Bedeutung (Osmostress) bei gleichermaßen mäßigen Tagesschwankungen der Osmolarität (DVO Daily Variation of Osmolarity) jedoch höheren Werten, die sich nicht mehr im physiologischen Durchschnittsniveau der Osmolarität befinden (330 $\mathrm{mOsmol} / \mathrm{L}$, orange $\mathrm{Li}$ nie). Die Farben der Pfeile spiegeln die maximalen (rot) und minimalen (grün) Werte einer einzelnen Schwankung wieder

\section{Osmolarität [mOsmol/L] and Osmostress}

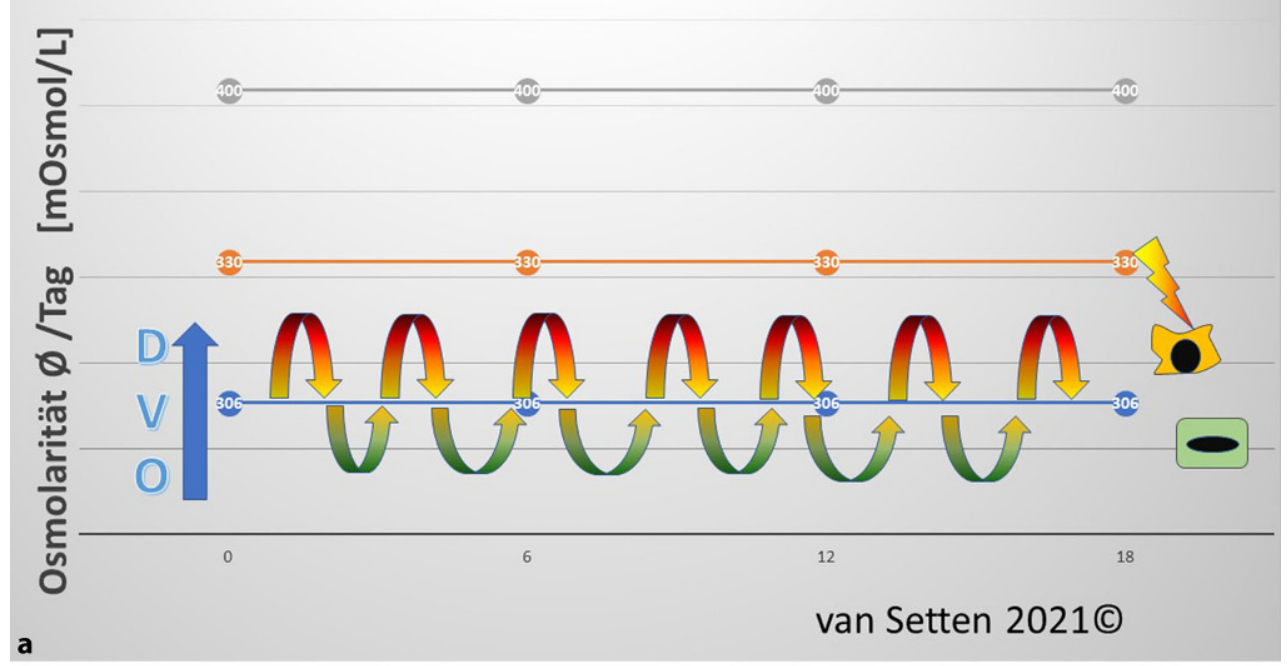

\section{Osmolarität [mOsmol/L] and Osmostress}

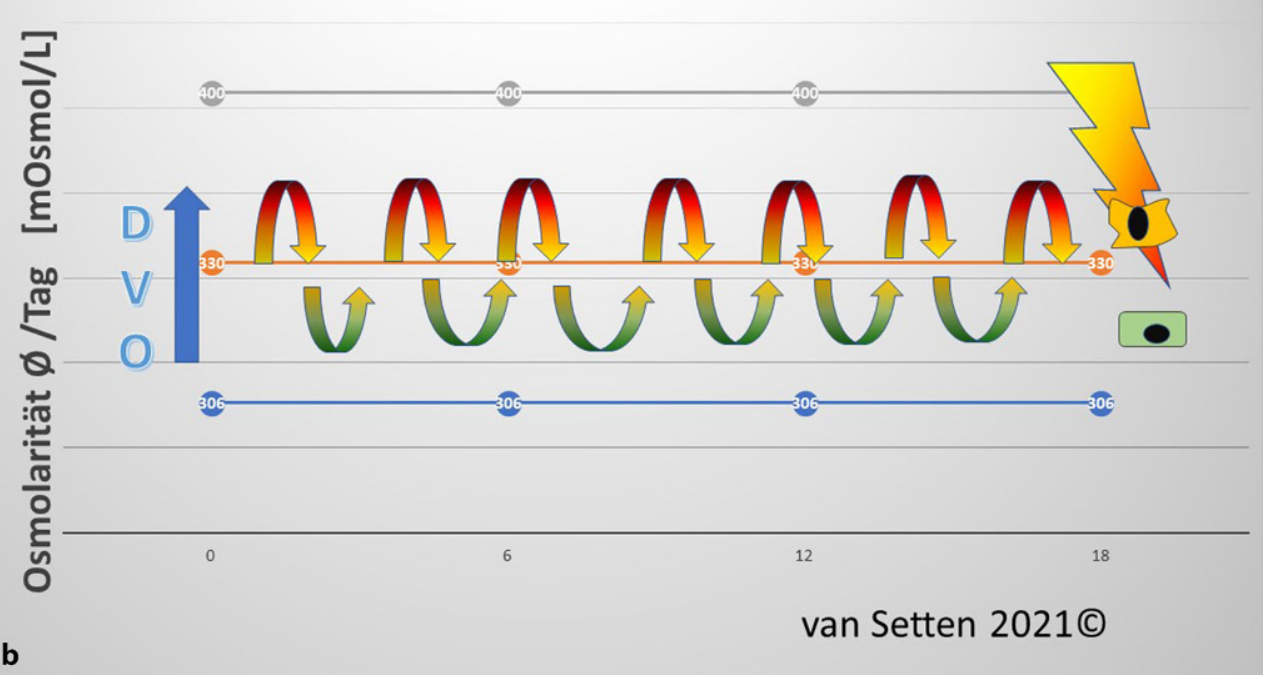


in ihrer Bedeutung für exponierte Zellen anscheinend vom allgemeinen Niveau der Osmolarität abhängig. Hyperosmolarer Stress führt zum Auftreten pro-inflammatorischer Zytokine wie tumor necrosis factoralpha (TNF-alpha), Interleukin-8, und Interleukin-6 [37]. Entzündungsmechanismen sind wiederum Teil jener Kernmechanismen, die eine Verschlechterung des Trockenen Auges als Krankheitzustand vorantreiben [38]. Es wurde die Hypothese aufgestellt [31], dass die pathogenetische Bedeutung der Schwankungen der Osmolarität mit dem allgemeinen Niveau der Osmolariät zunimmt (Abb. 6a, b). Interessanterweise scheinen Androgene den osmotischen Stress auf Epithelzellen zu vermindern und damit auch die hyperosmolaritätsbedingte Ankurbelung der Produktion von p-ERK, p-JNK, und p-p38 in exponierten Epithelzellen [37].

Therapeutisch sind daher im Model der Osmokinetik des Trockenen Auges eine langfristige und langsame, schonende Regulierung der Osmolarität und der Tageschwankungen ein Hauptziel der Behandlung.

Hierzu gehört auch die Verhinderung von iatrogenem osmotischem Stress, wie er zum Beispiel beim Gebrauch von hyper- oder hypoosmolaren Augentropfen auf die Augenoberfläche ausgeübt wird. Natürlich ist die pathophysiologische Bedeutung eines einzelnen Tropfens gering, aber als punktuelle Herausforderung der Homöostase erhöht sich der Effekt mit der Frequenz des Tropfens oder der Anzahl verschiedener Tropfen. Hier haben wir ein iatrogenes Osmolaritäts-JoJo [39], das in einem osmotischen Stress-Zirkel resultieren kann, der einer Wiedergewinnung eines Gleichgewichtes abträglich ist (Abb. 7). Ist das allgemeine Niveau oder die Stabilität dieses Niveaus, wie beim schweren Trockenen Auge schon vorab gestört, oder die Homöostase gefährdet, kann dieses zusätzliche iatrogene Osmolaritäts-Jojo die gerade noch erhaltbare Balance aus dem Geichgewicht bringen. Erste Ansätze mit leicht hypotonen Augentropfen zeigten positive Resultate und resultierten in einer Verminderung von Interleukin (IL)-6, IL-17 und Interferon (IFN) $-\gamma$ [40]. Eine optimierte Behandlung der Augenoberfläche sollte daher nicht nur konservierungsmittelfrei [41] sondern auch osmoneutral sein, d.h., die vorliegende Osmolarität nicht beeinflussend.

Ein Entzündungsgeschehen an und in der Augenoberfläche, wie auch mechanische Sensationen wie bei Attrition stimulieren nervale Reaktionsmechanismen [26] und haben damit eine hohe pathogenetische Relevanz in der Pathophysiologie des Trockenen Auges. Attrition wurde in diesem Zusammenhang definiert, als die, beim Trockenen Auge vorliegende, durch Schmierungsdefizit induzierte, gestörte mechanische Transduktion des Liddrucks auf die Augenoberflächen und deren sekundären Folgen, welche zu einer Dysregulation der Homöostase im Epithel mit Schmerzempfindung, Epithelschäden und sekundärer Entzündung führen kann. Die reibungs- und druckstimulierte Aktivierung von Neurorezeptoren [42, 43] kann dabei für das Aufkommen und den Erhalt einer neurogenen Komponente in der Entzündung der Augenoberfläche bei klinisch signifikantem Trockenen Augen eine bedeutende Rolle spielen. Dies kann eine der Ursachen sein, die für die hervorragende Wirkung von hochmolekularer Hyaluronsäure auf die Symptome bei Behandlung von schweren Trockenen Augen verantwortlich sind [30] - noch bevor eine signifikante Verbesserung klinischer Zeichen zu beobachten ist. Dieses hebt die Bedeutung des natürlichen, entzündungshemmenden Gleichgewichtssystems mit dem Vorkommen von CD44 als Hauptrezeptor für Hyaluronsäure an der Zelloberfläche [44, 45] und tumor necrosis factor-inducible gene 6 protein TSG-6 als kraftvoller antiinflammtorischer Faktor [46] und Mitglied der Hyaluronsäure-bindenden-Protein-Familie („Hyaladherine“) in der Pathophysiologie des Tro-

Abb. 7 latrogener Osmostress: Während iso-osmolare Augentropfen (blauer Tropfen) das Osmolaritäts-Gleichgewicht nicht wesentlich beinträchtigen, resultieren sowohl hyoposmolare Augentropfen (grüner Tropfen) als auch hyperosmolare Augentropfen (roter Tropfen) in einem deutlichen osmotischen Stress

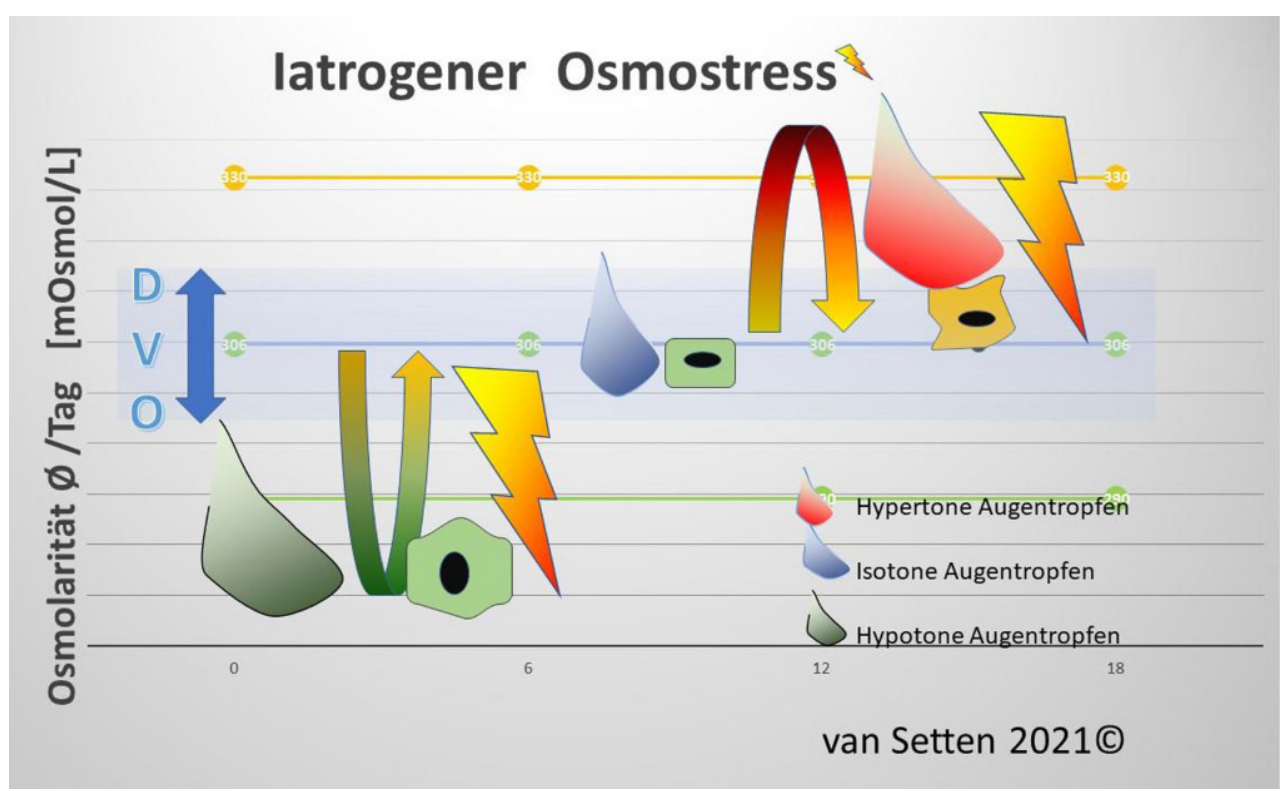


ckenen Auges hervor. Die genauen Wirkungs- und Regulationsmechanismen sind dabei vor allem für TSG-6, als erst kürzlich im Hornhautepithel vorkommend beschrieben [47], noch unklar, während CD44 dagegen ein schon gut untersuchter Rezeptor ist, dessen vermehrte Präsenz im konjunktivalen Gewebe von Trockenen Augen und dessen Bedeutung für die Pathophysiologie des Trockenen Auges schon länger bekannt ist [48]. Das Vorhandensein eines kraftvollen anti-inflammatorischen Wirkungsmechanismus im Hornhautepithel kann ein wichtiger Regulationsmechanismus der Augenoberfläche sein, der grundlegenden Prozessen einer Entwicklung, eben der Entzündung beim Trockenen Auge entgegenwirkt. Hier deuten sich neue und effektive Behandlungsoptionen an.

\section{Zusammenfassung}

Der Zustand der Augenoberfläche stellt besondere Anforderungen an das umgebende Schmiermedium, das heißt an den Tränenfilm. Es ist nicht nur die Zusammensetzung und Menge des Tränenfilms alleine, die über die Entwicklung einer Erkrankung des Trockenen Auges entscheiden, auch können beide Mangelzustände der Tränenflüssigkeit (qualitativ und quantitativ) über längere Zeit kaum deutlich voneinander getrennt werden. Es sind auch und vor allem die Anforderungen der Augenoberfläche an die Tränenfilmleistung, die von den mikro-tektonischen Eigenschaften, der $\mathrm{zu}$ bedeckenden und zu schützenden Oberfläche und der gleichzeitigen Umwelteinwirkung abhängen. Verschiedene chirurgische Eingriffe können zu (oft vorübergehenden) übermäßigen Schmieranforderungen führen, welche die physiologischen Tränenfilmeigenschaften überfordern können. Hier ist das Model der Attrition zu einem wertvollen Konzept geworden, um die daraus resultierenden pathophysiologischen Mechanismen zu verstehen. In diesem Modell spielt die Entzündung eine wichtige Rolle, die auch durch osmotischen Stress ausgelöst werden kann. Das Modell der Osmokinetik gestattet einen leichteren Zugang zu entscheidenden Variablen wie Tagesschwankungen, Frequenz etc. und deren Veränderungen über einen bestimmten Zeitraum. Das Modell der Osmokinetik schafft ein besseres Verständnis für die Pathophysiologie des Trockenen Auges als die Festlegung eines bestimmten absoluten Schwellenwertes. Die Identifizierung von zellulärem Stress als zentrales Thema in der Pathophysiologie von Erkrankungen des Trockenen Auges und seine dynamische Bedeutung könnten neue Perspektiven für die adaptive und optimierte Therapie des Trockenen Auges bieten.

\section{Conclusion}

Ocular surface characteristics impose specific requirements on its surrounding lubrication medium com- monly termed tear film. Alterations of the ocular surface impose a major challenge on the surrounding tear film to maintain adequate lubrication. It is not only the composition and quantity of the tear film that decides over the development of a dry eye disease, neither can both states of deficiency be strictly separated. It is also the ocular surface's demand on tear film performance, that is dependent on the micro tectonic characteristics of the surface to be covered and protected. Additionally simultaneous environmental conditions may challenge the tear film performance. Different surgical techniques can lead to excessive lubrication demands, that can exceed physiological tear film characteristics. Here the model of attrition has become a valuable concept to understand the resulting pathophysiological mechanisms. In this model inflammation plays a major role which can also be triggered by osmotic stress. The estimation of the intensity of osmotic stress has become easier accessible with the introduction of the model of osmokinetics, addressing more the changes of osmolarity over a certain time than a specific threshold value as in current models. The identification of cellular stress as a key issue in the pathophysiology of dry eye disease and its dynamic implication could offer new perspectives in adaptive and optimized therapy of dry eye disease.

Danksagung Der Autor dankt der Karin Sandqvists Stiftung (Karin Sandqvists Stiftelse) Stockholm, Schweden, der AugenStiftung (Ögonfonden), Stockholm, Sweden für die erhaltene Förderung und der Aviation-Ophthalmology Inc., Danderyd, Schweden für technische Hilfe bei der Gestaltung des Manuskriptes.

Funding Open access funding provided by Karolinska Institute.

\section{Einhaltung ethischer Richtlinien}

Interessenkonflikt G.-B. van Setten gibt an, dass kein Interessenkonflikt besteht.

Ethische Standards Es wurden keine spezifischen Untersuchungen am Menschen oder an menschlichem Gewebe durchgeführt, die eine Zustimmung der zuständigen Ethikkommission, im Einklang mit nationalem Recht sowie gemäß der Deklaration von Helsinki von 1975 (in der aktuellen, überarbeiteten Fassung) erfordert hätten.

Open Access Dieser Artikel wird unter der Creative Commons Namensnennung 4.0 International Lizenz veröffentlicht, welche die Nutzung, Vervielfältigung, Bearbeitung, Verbreitung und Wiedergabe in jeglichem Medium und Format erlaubt, sofern Sie den/die ursprünglichen Autor(en) und die Quelle ordnungsgemäß nennen, einen Link zur Creative Commons Lizenz beifügen und angeben, ob Änderungen vorgenommen wurden.

Die in diesem Artikel enthaltenen Bilder und sonstiges Drittmaterial unterliegen ebenfalls der genannten Creative Commons Lizenz, sofern sich aus der Abbildungslegende nichts anderes ergibt. Sofern das betreffende Material nicht unter der genannten Creative Commons Lizenz steht und die be- 
treffende Handlung nicht nach gesetzlichen Vorschriften erlaubt ist, ist für die oben aufgeführten Weiterverwendungen des Materials die Einwilligung des jeweiligen Rechteinhabers einzuholen.

Weitere Details zur Lizenz entnehmen Sie bitte der Lizenzinformation auf http://creativecommons.org/licenses/by/4. 0/deed.de.

\section{Literatur}

1. Dohlman TH, Lai EC, Ciralsky JB. Dry eye disease after refractive surgery. Int Ophthalmol Clin. 2016;56(2):101-10.

2. Naderi K, Gormley J, O'BrartD. Cataract surgery and dry eye disease: a review. Eur J Ophthalmol. 2020;30(5):840-55.

3. Quinto GG, Camacho W, Behrens A. Postrefractive surgery dry eye. Curr Opin Ophthalmol.2008;19(4):335-41.

4. Toda I. Dry eye after LASIK. Invest Ophthalmol Vis Sci. 2018;59(14):des109-des15.

5. Horwath-Winter J, et al. Influence of single-fraction Gamma-Knife radiosurgery on ocular surface and tear function in choroidal melanoma patients. $\mathrm{Br} \mathrm{J}$ Ophthalmol. 2013;97(4):466-70.

6. Garg P, et al. Dry eye disease after cataract surgery: study of its determinants and risk factors. Turk J Ophthalmol. 2020;50(3):133-42.

7. Clegg JP, et al. The annual cost of dry eye syndrome in France, Germany, Italy, Spain, Sweden and the United Kingdom among patients managed by ophthalmologists. Ophthalmic Epidemiol.2006;13(4):263-74.

8. McDonald M, etal.Economic and humanistic burden of dry eye disease in Europe, North America, and Asia: a systematic literature review. Ocul Surf. 2016;14(2):144-67.

9. Jones MB, et al. Elastohydrodynamics of the eyelid wiper. Bull Math Biol.2008;70(2):323-43.

10. CraigJP, etal.TFOSDEWSII report executivesummary. Ocul Surf. 2017;15(4):802-12.

11. van Setten G. The anatomical dry eye-a different form of ocular surface disease deserves focus. OJOph. 2017;7:184-90.

12. Steven $P$, et al. Influence of aging on severity and antiinflammatory treatment of experimental dry eye disease. Klin MonblAugenheilkd. 2017;234(5):662-9.

13. Baudouin $\mathrm{C}$, et al. Revisiting the vicious circle of dry eye disease: a focus on the pathophysiology of meibomian gland dysfunction. BrJOphthalmol. 2016;100(3):300-6.

14. Heegaard S, Knudsen LL, van Setten G, Koranyi G, Holopainen JM, Kaarinranta K, Klyve P, Raeder S. Nordic guidelines dry eye disease. : Dansk Oftalmologisk Selskab Archives; 2016. S. 26.

15. Kaercher T, Bron AJ. Classification and diagnosis of dry eye. Dev Ophthalmol.2008;41:36-53.

16. Horwath-Winter J, et al. Evaluation of the clinical course of dry eye syndrome. Arch Ophthalmol. 2003;121(10):1364-8.

17. Bron AJ, Evans VE, Smith JA. Grading of corneal and conjunctival staining in the context of other dry eye tests. Cornea. 2003;22(7):640-50.

18. Hamrah P, et al. Optimizing evaluation of Lissamine Green parameters for ocular surface staining. Eye (Lond). 2011;25(11):1429-34.

19. Baudouin C. A new approach for better comprehension of diseases of the ocular surface. J Fr Ophtalmol. 2007;30(3):239-46.

20. Daull P, et al. Ocular surface response of two preservativefree cylcosporine A emulsion eye drops in a mouse model of dry eye. Curr Eye Res. 2021;46(8):1096-1104. https:// doi. org/10.1080/02713683.2021.1878228.
21. Baudouin C, et al. One-year efficacy and safety of $0.1 \%$ cyclosporine a cationic emulsion in the treatment of severe dry eye disease. Eur J Ophthalmol. 2017;27(6):678-85.

22. Periman LM, et al. The immunological basis of dry eye disease and current topical treatment options. J Ocul Pharmacol Ther. 2020;36(3):137-46.

23. van Setten G, et al. Evidence of seasonality and effects of psychrometry in dry eye disease. Acta Ophthalmol. 2016;94(5):499-506.

24. Perez VL, Stern ME, Pflugfelder SC. Inflammatory basis for dry eye disease flares. Exp Eye Res. 2020;201:108294.

25. van Setten GB, Mueller-Lierheim W, Baudouin C. Dry eye etiology: focus on friction. Klin Monbl Augenheilkd. 2020;237(10):1235-1236. https://doi.org/10.1055/a-08983857.

26. van Setten GB. Impact of attrition, intercellular shear in dry eye disease: when cells are challenged and neurons are triggered. Int J Mol Sci. 2020;21(12):4333. https://doi.org/ 10.3390/ijms21124333.

27. Bron AJ, Willshire C. Tear osmolarity in the diagnosis of systemic dehydration and dry eye disease. Diagnostics. 2021;11(3):387. https://doi.org/10.3390/ diagnostics 11030387.

28. Akpek EK, et al. Dry eye syndrome preferred practice pattern. Ophthalmology. 2019;126(1):P286-p334.

29. Yazdani M, et al. Tear film break-up time and dry eye disease severity in a large Norwegian cohort. J Clin Med. 2021;10(4):884.https://doi.org/10.3390/jcm10040884.

30. van Setten GB, et al. The HYLAN M study: efficacy of $0.15 \%$ high molecular weight hyaluronan fluid in the treatment of severe dry eye disease in a multicenter randomized trial. J Clin Med. 2020:9(11):3536. https://doi.org/10.3390/ jcm9113536.

31. van Setten GB. Osmokinetics: defining the characteristics of osmotic challenge to the ocular surface. Klin Monbl Augenheilkd.2020;237(5):644-8.

32. Finan JD, Guilak F. The effects of osmotic stress on the structure and function of the cell nucleus. J Cell Biochem. 2010;109(3):460-7.

33. Finan JD, Leddy HA, GuilakF. Osmotic stress alters chromatin condensation and nucleocytoplasmic transport. Biochem Biophys Res Commun. 2011;408(2):230-5.

34. CalanceDN, etal. Hypertonicity primes malignant melanoma cells for apoptosis. Apoptosis. 2018;23(3-4):201-9.

35. Rossi C, et al. Cell stress induces mislocalization of transcription factors with mitochondrial enrichment. Int J Mol Sci. 2021;22(16):8853. https://doi.org/10.3390/ ijms22168853.

36. Bonny AR, et al. Stress-induced growth rate reduction restricts metabolic resource utilization to modulate osmoadaptation time. Cell Rep. 2021;34(11):108854.

37. Zibandeh N, et al. Androgen suppresses hyperosmolarityinduced inflammatory mediators in human corneal epithelial cells. Cornea. 2020;39(7):886-91.

38. Baudouin C, etal. Clinical impact ofinflammation in dry eye disease: proceedings of the ODISSEY group meeting. Acta Ophthalmol.2018;96(2):111-9.

39. vanSetten GB. Osmokinetics: a new dynamic conceptin dry eye disease. J Fr Ophtalmol. 2019;42(3):221-5.

40. Li Y, et al. Comparison of $0.3 \%$ hypotonic and isotonic sodium hyaluronate eye drops in the treatment of experimental dry eye. Curr Eye Res. 2017;42(8):1108-14.

41. Gomes JAP, et al. TFOS DEWS II iatrogenic report. Ocul Surf. 2017;15(3):511-38.

42. Belmonte C, et al. TFOS DEWS II pain and sensation report. OculSurf. 2017;15(3):404-37. 


\section{themenschwerpunkt}

43. Belmonte C, García-Hirschfeld J, Gallar J. Neurobiology of ocular pain. Prog Retin Eye Res. 1997;16(1):117-56.

44. Mattheolabakis G, et al. Hyaluronic acid targeting of CD44 for cancer therapy: from receptor biology to nanomedicine. J Drug Target. 2015;23(7-8):605-18.

45. Misra S, et al. Hyaluronan-CD44 interactions as potential targets for cancer therapy. FEBS J. 2011;278(9):1429-43.

46. Wisniewski HG, Vilcek J. TSG-6: an IL-1/TNF-inducible protein with anti-inflammatory activity. Cytokine Growth Factor Rev. 1997;8(2):143-56.

47. Lardner E, van Setten GB. Detection of TSG-6-like protein in human corneal epithelium. Simultaneous presence with
CD44 and hyaluronic acid. J Fr Ophtalmol. 2020. https:// doi.org/10.1016/j.jfo.2020.03.005.

48. Brignole F, et al. Efficacy and safety of $0.18 \%$ sodium hyaluronate in patients with moderate dry eye syndrome and superficial keratitis. Graefes Arch Clin Exp Ophthalmol. 2005;243(6):531-8.

Hinweis des Verlags Der Verlag bleibt in Hinblick auf geografische Zuordnungen und Gebietsbezeichnungen in veröffentlichten Karten und Institutsadressen neutral. 